\title{
Enfermedad de Kawasaki
}

\author{
Dra. Carmen Avilés $\mathbf{L}^{3}{ }^{3}$; Dra. Jimena Guerrero R. ${ }^{1,2}$; Dra.. Erna Lueg. F. ${ }^{3}$; \\ Dra. Pía Spalloni M. ${ }^{1}$; Dr. Carlos Toro A. ${ }^{2,3}$.
}

\section{Kawasaki disease}

\begin{abstract}
Among 5399 children admited to the pediatric wards of a Metropolitan hospital at Santiago, Chile. from January 1984 to December 1986, 6 cases of confjmed Kawasaki disease were detected: all showed fever, cutaneous eruption and ocular and oropharyngeal enantema. Five showed the typical distal lesions at the limbs. Limph node enlargement was also evident in 5 children. Age at the begining of symptoms varied from 8 months to 10 years. Three patients were females. Three children (two boys and one girl) showed cchographic evidence of coronary artery involvement, in one of them abnormal Q waves were seen. in the electrocardiogram (ECG) at the first month of follow up. Abnormal $Q$ waves were al so present at the end of the first month in a fourth patient without echocardiographic signs of coronary lesions. In both cases there were no symptoms attributable to miocardial infarction and normal ECG were recorded 7 and 9 months later respectively. Coronary abnormalities occurred in spite of early standard treatment schedule with acetylsalicylic acd. No cases of meningeal involvement occurred among our cases but one patient had clinical and electroencephalographic signs suggestive of severe reduction of neuronal activity during the acute stage that later dissappeared without neurological sequelae.

(Key words: Kawasaki disease).
\end{abstract}

La enfermedad de Kawasaki o síndrome linfomucocutáneo es una vasculitis aguda febril de infancia, de causa desconocida, descrita en 1967 en Japón por dicho autor. Posteriormente se ha detectado en casi todas las regiones y razas del mundo ${ }^{1-3}$. Sus manifestaciones principales son: fiebre prolongada, conjuntivitis no exudativa bilateral, exantema polimorfo, linfadenopatías cervicales, cambios en las extremidades incluyen. do eritema palmo plantar con descamación y alteraciones bucales, caracterizadas por lengua fresa, labios rojos fisurados y enantema orofaríngeo ${ }^{1}$.

La afección ocurre predominantemente en niños entre 2 meses y 4 affos de edad, pero $15 \%$ de los casos suceden en niños mayores. Su diagnóstico es clínico y requiere la presencia de por lo menos cinco de las seis manifestaciones recién

1 Servicio de Pedialría, Unidad de Infecciosos. Hospital Prula Jaraquemada.

2. Departamento de Pediatria, División Ciencias Médicas Sur. Facultad de Medicina de La Universidad de Chile.

3. Servicio de Pediatría, Unidad de Cardiología. Hospital Paula Jaraquemada. descritas. No hay exảmens de laboratorio patogno. mónicos pero la trombocitosis en la sangre periférica se considera un hecho relevante para su reco. nocimiento.

La importancia de la enfermedad radica en que provoca secuelas cardjovasculares, principalmente aneurismas de las arterias coronarias y otros territorios, que se detectan en una proporción śignificativa de los casos y pueden sufrir complicaciones como rotura, trombosis, infartos, disfunción de músculos papilares, compromiso miocárdico y pericárdico, produciendo una letalidad del orden de $1,4 \%$.

Con el propósito de destacar las manifestaciones clínicas, los hallazgos de laboratorio, los resultados de los estudios cardiovasculares en esta afección y estimular el interés para buscarla, presentamos seis casos estudiados en un servicio de pediatria. No hemos encontrado descrito el síndrome en la literatura nacional.

\section{PACIENTE Y METODO}

Entre enero de 1984 y diciembre de 1986, se hospitalizaron 5.399 niños en el Servicio de Pediatría del Hospital Paula Jaraquemada, seis cumplian con al menos cinco de los seis criterios clínicos para el diagnós. 
twoo de Enfermedad de Kawasaki ${ }^{1}$. Tres tenian además otras afecciones de origen congénito isíndrome de Down, sindrome de Holt Oran y macrosomía familiar, respectivamente).

En todos los pacientes se realizó anamnesis dirigida, examen físico completo y exámenes de laboratorio seriados incluyendo hemograma, vclocidad de eritrosedimentación (VHS), recuento de plaquetas; cuiltivos bacteriológicos de sangrc, orina y deposiciones; exámenes parasitológicos seriados de deposiciones: rcaccioncs de aglutinación (Widal). reacciones de Paul Bunell Davidsohn, proteína C reactiva, antiestreptolisinas 0 , creatinina, nitrógeno ureico, transaminasas, bilirrubina iosfotasas alcalinas, estudio de inmunoglobulinas $y$ com. plemento en la sangre; examen completo de orina: radiografías de tórax; ecocardiogratías y clectrocardiogramas.

En la mayoria de los pacientes se hicieron también exámenes y cultivos de líquidos eefaloraquídeo (LCR), mielocultivos, búsqueda de células de lupus, factor reumatoídeo y radiografías de cavidades cardíacas. En un paciente, que además, tenía anemia hemolítica autoinmune, se realizaron recuentos de linfocitos $\mathbf{T}$ y $B$. pruebas de Coombs seriadas, anticuerpos calientes lgG, anticuerpos antipenicilina, estudio de facocitosis de levaduras y de nitroazul de tetrazolio.

- Todos los pacientes recibieron aspirina, la mayoria en dosis de $30 \mathrm{mg} \times \mathrm{kg} \times$ día con sólo dos excepciones (5 y $15 \mathrm{mg} \mathrm{c} \mathrm{kg} \mathrm{x} \mathrm{dia} \mathrm{respectiyamente)} \mathrm{de} \mathrm{acucrdo} \mathrm{con}$ criterios actualmente aceptados $5^{2,4,5}$. Todos habían recibjdo antibjóticos antes del diagnóstico definutivo.

\section{RESULTADOS}

A.lgunas características generales de los pacientes se expresan en la tabla. La edad fluctuó entre 8 meses y 10 años; cinco niños tenian menos de 20 meses al consultar, $y$ tres menos de 1 año. Tres pertenecian al sexo masculino.

Tres episodios se presentaron en invierno, dos en primavera y uno en otorio. Todos tuvieron fiebre que duró entré 13 y 32 días $(\bar{x} 22 \pm \mathrm{DE}$ 8,6 dias). Durante la evolución del sindrome febril, todos presentaron erupción cutánea polimorfa y eritema morfiliforme. En todos los casos hubo enrojecimiento conjuntival sin secreción purulenta; eritema, encostramiento y ulceración labial; lengua fresa y enantema faríngeo. En 5/6 casos se observaron lesiones en las extremidades, caracterizadas por edema palmar y plantar, enrojecimiento y descamación de los dedos y ortejos. En el mismo número de pacientes se palparon ganglios cervicales de tamaño aumentado. Debe ser destacado que la erupción cutánea fue recurrente en $4 / 6$ casos, motivando incluso la rehospitalización en dos pacientes: uno en tres oportunidades, la última siete meses después de la primera.
Otros hallazgos comunes fueron piuria estéril en $4 / 6$ pacientes; diarrea en $3 / 6$; artralgias en $3 / 6$ casos; compromiso del sistema nervioso central en $5 / 6$ casos, caracterizado por irritabilidad en 4 y obnubilación y sopor en 1 ; en ninguno se detectaron signos de irritación meníngea. En 5/6 casos habian síntomas respiratorios, principalmente tos y estertores bronquiales. No se detectaron síntomas ni signos clínicos de compromisó cardiovascular en 5 pacientes: en el otro (caso 3) se palpó un aneurisma axilar izquierdo a los 27 días de evolución.

La velocidad de eritrosedimentación (VHS) fue mayor que $20 \mathrm{~mm}$ en la primera hora al ingreso de todos bs pacientes (rango 24 a $100 \mathrm{~mm} \bar{x}$ $51,1 \pm$ DE $30 \mathrm{~mm}, 1^{\text {a hora) }}$.

Las pruebas de función hepática y renal fueron normales en todos los pacientes. Ningún caso tuvo ictericia.

En 5 niños habia discreta anemia normocitica y normocrómica, uno de ellos (caso 1) tuvo además signos significativos de hemólisis; como se señala más adelante. La cantidad de leucocitos en la sangre periférica fue mayor que $10.000 \mathrm{x}$ $\mathrm{mm}^{3}$ en $4 / 6$ pacientes fluctuando entre 5.200 y 15.400 ( $\mathrm{x} 11.650 \pm$ DE 4.0). En todos los casos habia desviación izquierda en el hemograma inicial $(\bar{x} 1.164$, rango 410 a 3.350 baciliformes $x$ $\mathrm{mm}^{3}$ ). El recuento de plaquetas demostró trom. bocitosis en $5 / 6$ pacientes, oscilando entre entre 490.000 y 1.070 .000 por $\operatorname{mm}(\bar{x} 692.000 \pm \mathrm{DE}$ 257.332). Sus elevaciones máximas se produjeron en dos casos en la segunda semana de evolución y en los restantes durante la $3^{a}, 5^{a}$ y $6^{a}$ semanas respectivamente. $\mathrm{La}$ paciente en que no se detectó aumento de las plaquetas (caso 5) fue la primera de la serie y sólo se le tomó una muestra, al comienzo de la enfermedad.

Se determinó proteína $\mathrm{C}$ reactiva en 5 paciente, resultando elevada en 3 . Se midieron concentraciones en $\mathrm{IgG}$ e IgM en $5 / 6$ pacientes, en dos ambas estaban elevadas, en uno aumentaron las IgG y en otro sólo las IgM. En 4/6 niños afectados se encontró piuria transitoria de más de 25 leucocitos por $\mu \mathrm{l}$ con urocultivos negativos. No se encontró albuminuria.

$\mathrm{La}$ investigación de anticuerpos antinucleares dio resultados negativos en todos los casos. Igualmente las reacciones de Widal, Paul Bunell Davidsohn, antiestreptolisinas 0 y cultivos bacteriológicos. El complemento fue nomal en todos $y$ el factor reumatoídeo fue positivo en $3 / 5$ 
sujetos en que se estudió.

En 4/6 pacientes se hicieron punciones lumbares, obteniếndose LCR de características quimico citológicas normales en todos; en el pacien. te con mayor compromiso sensorjal se realizó electroencefalograma que mostró depresión severa de la actividad neuronal, indicando compromiso generalizado cortical y subcortical. Las radiografías de tórax fueron normales en dos pacientes (uno con sintomas respiratorios). De los otros 4 pacientes, todos con síntomas, dos tenian infiltrados insterticiales, uno bronconeumonia y otro combinación de ambos tipos infiltrados.

En tres pacientes (casos 1, 3 y 5) los ecocar. diogramas demostraron alteraciones coronarias: en el caso 1, las ecocardiografias del primer mes de evolución mostraban dilatación fusiforme de la coronaria izquierda, pero nueve meses después tenja un aneurisma en el mismo yaso, a pesar de haber recibido aspirina en dosis de $30 \mathrm{mg} \mathrm{x} \mathrm{kg} \mathrm{x}$ día injeialmente $y$ luego $10 \mathrm{mg} \times \mathrm{kg} \times$ dia por 7 meses; en él no se han detectado alteraciones electrocardiográficas. El caso 3, el mismo paciente con aneurisma axilar izquierdo, tenía aneurismas que afectaban el origen de ambas arterias coronarias principales al mes de evolución, siendo el de la derecha casi del mismo diámetro de la aorta; en su electrocardiograma habian ondas $\mathbf{Q}$ de más de $4 \mathrm{~mm}$ en $V_{L}, V_{F} y_{4} V_{6} V_{6}$, sin alteraciones de la repolarización ventricular ni evidencia clínica de infarto, pero con fracción de acortamjento del ventrículo izquierdo algo disminuida. En este niño los aneurismas habían disminuido al cabo de siete meses y; dos años más tarde, el electrocardiograma era normal. En el caso 5 la ecocardiografía mostó, tres meses después del ingreso, dilatación leve fusiforme de la coronaria izquierda con engrosamiento de su pared, que persistían después de dos artos de seguimiento, sin alteraciones electrocardiográficas. En $2 / 3$ pacientes con lesiones coronarias detectables la duración de la fiebre fue mayor de 14 días, lo misno puede decirse del paciente que mostró ondas $Q$ patológicas al comienzo de la enfermedad, sin alteraciones ecográficas en esos vasos (tabla).

En los restantes pacientes (3/6) los electrocardiogramas fueron normales en 2 (excepto por taquicardia sinusal); en el tercero (caso 6) habían ondas $Q$ de más de $10 \mathrm{~mm}$ en $V_{5}$ y $V_{6}$ que desaparecieron 15 meses después: en este caso

Tabla

Características generales en 6 niños con enferptedad de Kawasaki

\begin{tabular}{|c|c|c|c|c|c|c|c|c|}
\hline $\mathrm{N}^{\circ}$ & $\begin{array}{l}\text { Caso } \\
\text { Sexo }\end{array}$ & $\begin{array}{c}\text { Edad } \\
\text { (meses) }\end{array}$ & $\begin{array}{l}\text { Fiebre } \\
\text { duración: } \\
\text { (días) }\end{array}$ & $\begin{array}{l}\text { Plaquetas } \\
\text { máximo } \\
\times \mathrm{mm}^{3}\end{array}$ & $\begin{array}{l}\text { Electro-CG } \\
\text { (mes } \\
\text { evolución) }\end{array}$ & $\begin{array}{l}\text { Eco-2 D } \\
\text { (mes } \\
\text { evolución) }\end{array}$ & $\begin{array}{l}\text { Recidi- } \\
\text { vas }\end{array}$ & $\begin{array}{l}\text { Enfermedad } \\
\text { asociada }\end{array}$ \\
\hline 1 & M & 8 & 30 & 510.000 & Normal & (lm) D:CI & $\mathrm{Si}$ & $\begin{array}{c}\text { Macrosomia } \\
\text { AHAl }\end{array}$ \\
\hline 2 & $\mathrm{~F}$ & $\mathrm{p}$ & 14 & 1.070 .000 & Normal & Normal & No & No \\
\hline 3 & M & 20 & 32 & 540.000 & $\begin{array}{c}\text { (1m) } Q: \mathbf{l}, \mathrm{II}, \mathrm{L}, \mathrm{F} \\
4-6 \\
(7 \mathrm{~m}) \text { Normal }\end{array}$ & $\begin{array}{c}\text { (1m) A: CIy CD } \\
\quad \downarrow \text { Fac. } \\
\text { (7m) Normal }\end{array}$ & Sí & No \\
\hline 4 & $\mathrm{~F}$ & 8 & 16 & 850.000 & Normal & Normal & Sí & No \\
\hline 5 & $\mathrm{~F}$ & 120 & 13 & $114.000^{*}$ & Normal & $\begin{array}{l}\text { (1m) } \uparrow \text { Pared CI } \\
(13 m) D: C I\end{array}$ & I Sí & Trisomía 21 \\
\hline 6 & M & 18 & 27 & 490.000 & $\begin{array}{l}\text { (m) Q: 4-6 } \\
\text { (9m) Normal }\end{array}$ & Normal & No & Holt-Oram \\
\hline
\end{tabular}

D: Dilatación fusiforme, A: aneurisma. CI: tronco atteria coronaria izquierda. CD: arteria coronaria derecha. DA: arteria coronaria descendente anterior. Fac: fracción de acortamiento. $\downarrow$ : disminución. Taumento AHAI: anemia hemolítica autoinmune. Q:ondas q anormales para la edad. *En este caso se realizó sólo un recuento al comienzo de los síntomas. 
tampoco se documentaron alteraciones significativas en la ecocardiografía. Sólo en un paciente se observaron alteraciones ecocardiográficas de la función miocárdica: correspondia a] niño con aneurismas de ambas coronarias (caso 3 ).

Lamentablemente no fue posible realizar determinaciones enzimáticas simultáneas en los enfermos con alteraciones electrocardiográficas, pero ninguno tuvo signos de insuficiencia cardiaca, pericarditis o insuficiencia mitral.

Uno de estos pacientes (caso 1) presentó, junto con los signos de enfermedad de Kawasaki, anemia hemolítica autoinmune con anticuerpos calientes IgG positivos, anticuerpos antipenicilina en concentraciones bajas (1/2) y reacción de Coombs directa positiva que persistió durante 5 meses para desaparecer 8 meses después del comienzo de sus síntomas.

\section{DISCLSION}

A pesar de los esfuerzos de muchos investigadores, la causa del sindrome de Kawasaki perma. nece desconocida; la tendericia actual se inclina hacia la busqueda de un agente infeccioso por tratarse de una enfermedad febril, autolimitada, exantemática, semejante a otras enfermedades infecciosas. La hipótesis más espectacular se refiere al rol que juega un ácaro, el Dermatophagoides farinae, en la patogénesis de la enfermedad, ya sea a través de una respuesta inmune a antigenos del ácaro o bien de la acción de éste como vector para algún agente infeccioso, similar a las richettsias, como el propionibacterium acness, que ha sido identificado en su tubo diges tivo y se parece a algunas estructuras encontradas en los ganglios binfáticos, bazo y piel de pacientes afectados. Se ha postulado también una respuesta de hipersensibilidad a uno o más antigenos es. treptocócicos, lo que no ha sido confirmado hasta ahora. 9,10

Cuatro de nuestros 6 pacientes presentaron las seis manifestaciones clínicas principales, sólo en uno no se detectaron las adenopatias (que ocutren en $70 \%$ de los casos) y en otro faltaron las alteraciones de las extremidades (descritas en 76 a 94\%), de modo que todos cumplian con los criterios internacionales para el diagnóstico ${ }^{1}$.

Se han descrito una serie de hallazgos clínicos asociados a este síndrome que demuestran el compromiso multisistémico que produce ${ }^{1,11,12}$.
Las alteraciones-del sistema nervioso central en nuestros pacientes, fueron moderadas y caracte. rizadas principalmente por irritabilidad o trastorno leve de conciencia, peto no encontramos casos con sîndrome meníngeo, ni alteraciones del liquido cefalorraquideo, como se describe hasta en $50 \%$ de los afectados en otras series ${ }^{1} 1$. El niño que sufrio mayor compromiso de conciencia, presentaba alteraciones electroencefalográficas que'sugerían compromiso encefálico, atribuible tal vez a la vasculitis que caracteriza el proceso, hecho no suficientemente subrayado en la literatura que, al referirse a las complicaciones neurológicas de la enfermedad, menciona preferentemente la meningitis aséptica, excepto en el brote de New England ${ }^{6}$. Convendría tener presente que estos pacientes pueden tener encefalitis con escaso compromiso meningeo, como parece haber ocurrido en este niño. La frecuencia de artralgias y diarrea (50\% de los casos) es mayor que la descrita por otros autores: no sabemos si se debe a diferencias étnicas o al escaso número de pacientes; lo mismo ocurre con la frecuencia con que detectamos infiltrados pulmonares que alcanzó a 4/6 pacientes.

Se ha señalado que la piuria aséptica es causada aparentemente no sólo por arteritis sino también de origen renal y desaparece en la convalescencia ${ }^{1,9}$.

Si bien la mayoría de nuestros pacientes tuvo taquicardia en algún momento de su evolución, no sufr ieron manifestaciones clínicas de compromiso pericárdico, endocárdico o miocárdico, no obstante lo cual tres tenían, después del primer mes de evolución, dilataciones fusiformes o francos aneurismas coronarios y en uno de ellos las lesiones evolucionaron en ocho meses desde dilatación fusiforme a aneurisma, ilustradndo la posibilidad de progresión arrastrada, señalada por otros. En otro paciente las lesiones coronarias de la ecografía se acompafiaron de disminución de la función ventricular y alteraciones electrocardiográficas sugerentes de isquemia, todas las cuales, afortunadamente habían desaparecido al cabo de ocho meses. A pesar de no contar aún con un seguimiento sistemático, la evolución de nuestros pacientes ilustra las dificultades para predecir el curso que asumian en cada caso de las lesiones coronarias, que puede aún tener diferencias raciales. En efecto, estudios recientes en Japón con arteriografía coronaria demostraron que $22 \%$ de los pacientes tenía aneurismas en los 
tres primeros meses de cvolución, desapareciendo $54 \%$ de ellos entre 6 y 24 meses después, regresión que puede comenzar antes de los tres meses de iniciada la enfermedad4,12. En cambio, en una población racialmente diferente la persistencia de lesiones coronarias era del orden de $80 \%$ al cabo de 3 años, no observándose regre. siones significativas posteriorés al $9^{\circ}$ mes de seguimiento ${ }^{2}$. Como en las series mayores de pacientes, también en ésta las lesiones coronarias más significativas se detectaton en los niños en quienes la fiebre tuvo mayor duración ${ }^{13}$.

La única explicación que tenemos para la anemia hemolítica de uno de nuestros pacientes, es que se trate de una simple coincidencia entre una reacción inmune, precipitada por la penicilina (ya que se encontraron anticuerpos circulantes contra la droga) y la enfermedad de Kawasaki.

El tratamiento del síndrome de Kawasaki, se puede dividir en dos etapas. En la fase aguda se recomienda actualmente el empleo de aspirina en dosis que fluctúan entre 30 y $100 \mathrm{mg} \times \mathrm{kg} \times$ día, continuado después de la normalización de la temperatura y de las velocidad de sedimentación con $10 \mathrm{mg} x=\mathrm{kg} x$ dia durante un minimo de tres meses e indefinidamente si existen evidencias de lesiones coronarias. No se ha probado que este tratamiento disminuya la frecuencia de alteraciones coronarias, pero sí de la obstrucción posterior de las mismas. Más recientemente se ha demostrado que la administración de gamaglobulina endovenosa, $400 \mathrm{mg} \times \mathrm{kg}$ x día durante 5 dias, asociada con aspirina en las dosis señaladas, reduce significativamente la prevalencia de anomalías coronarias cuando es suministrada temprano en el curso del sindrome, especialmente los aneurismas gigantes y por ende de infartos de miocardio, cuya ocurnencia se asocia estrechamente con los primeros $5,8,14-15$. La gran limitación de este tratamiento reside en su elevado costo.

Afortunadamente no se conocen otras complicaciones de la enfermedad que no sean las anomalías vasculares descritas, pero no debe olvidarse que la afección puede presentar recu. rrencias que, en nuestra serie ocurrieron hasta 7 meses después del primer brote. En todo caso el seguimjento regular de estos pacientes está formalmente indicado con controles ultrasonográ. ficos mientras persista evidencia de lesiones arteriales coronarias.

\section{RESUMEN}

Entre 5.399 niños ingresados entre enero de 1984 y diciembre de 1986 a un servicio de pediatria de un hospital metropolitano de Santiago, 6 cumplían los criterios de diagnóstico para la enfermedad de Kawasaki; su edad al comienzo de los síntomas variaba entre 8 meses y 10 años, 3 eran varones; la fiebre duró entre 13 y 32 días, todos tuvieron enupción cutánea, enantema bucal y faringeo $\mathbf{e}$ inyección conjuntival, 5 tuvieion lesiones en las extremidades y en 5 se detectaron adenopatias. La erupción cutánea fue recurrente en 4 pacientes, uno de los cuales sufrió 3 recidivas, la última 7 meses đespués de la primera hospitalización. Tres niños afectados mostraron evidencia ecográfica de lesiones coronarias $y$, aunque ninguno tuvo clara evidencia clínica de infarto, uno tenia además reducción de la fracción de acortamiento de ventriculo izquierdo $y$ ondas $Q$ anormales en el electrocardiograma, las que también fueron detectadas en un cuarto paciente sin anomalias ecográficas coronarias. Otro niño presento simultáneamente signos de anemia hemolítica por anticuerpos calien. tes IgG y anticuerpos antipenicilina en concentra. ciones bajas. Todos los niños afectados fueron tratados con aspirina en las dosis recomendadas para este efecto. Ninguno de nuestros pacientes mostró signos clínicos de irritación meníngea pero uno de ellos tuvo mareado compromiso de conciencia y su electroencefalograma mostró severa depresión neuronal, recuperándose completamente más tarde.

\section{REFERENCIAS}

1. Kawaseki T.: Clinical features of Kawasaki syndrome. Acta Paediatr Jpn 1983; 25: 79-88.

2. Tumer-Gomes S., Rose V., Bresina A., Smalhorn, Rowe $R_{\text {.: }}$ High persistence rate of established coronary artery lesions secondary to Kawasaki disease among a panethnic Canadian population. J. Pediatr 1986; 108: $928 \cdot 932$.

3. Solo E.t Pelkonen P., Pettay O.: Outbreak of Kawasaki syndrome in Finland. Acta Paediatr Scand 1986 ; 75 : 75-8-

4. Kusakava $S$.: Long tem administrative care of Kawasaki disease. Acta Paediatu Jpn 1983; 25: 205-208.

5. Furuso K., Kamiya T, Nakano H, y cols.: High dose intravenous gammaglobulin for Kawasakì disease. Lancet 1982;2: 1055 .

6. Maede R.H. Brandt L.: Manifestations of Kawasaki disease in New England outbreak. I Pediatr 1982; 100: 558-562. 
7. Newburger J., Takahashi M., Burns S. etal: The treatment of Kawasaki syndrome with intravenous gammaglobulin. N Eng J Med 1986: 315: 341-347.

8. Negeshima M., Matsushima, M., Marsuola H., Ogawa $A .$, Okumura $N$.: High dose gammaglobulin therapy for Wawasaki disease. J Pediat 1987; 110:710.712.

9. Hanashima Y., Tasaka K., Fujiwara H., Hoshivo T. Matsuda S., Kao T.: Kawasaki's disease. Its pathological feature and possible pathogenesis. Acta Paediatr Jpn 1983: 25: 108-117.

10. Yamamoto T.: Etilogy of the mucocutanous lymph node syndrome, and the Streptococcus hypothesis. Acta Paediatr Jpn 1983; 25: 118-126.

11. Ohtsuka Ch., Watanabe K., Honda $T$, et al: Complicaction of the central nervous system in Kawasaki disease. Acta Paediatr Jpn 1983; 25: 176-179.
12. Kato $H$., Ichinose E.; Cardiovascular involvement in Kawasaki disease. Acta Paediatr Jpn 1984; 24: 132-145.

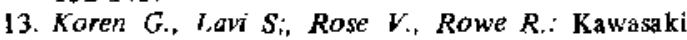
disease: Review of risk factor for coronary aneurismas. J Pediatr 1986; 108: 256-259.

14. Kohr R.: Progressive asymptomatic coronary artery disease as a tate fatal sequela of Kawasaki disease. J Pediats 1986; 108: 256-259.

15. Canter Cht, Bower R., Struss A.: Enfermedad de Kawasaki atípica con aneurísma aórtico. Pediatrics (esp) $1981 ; 12: 457.459$.

16. Nakano H., Saito H., Vedo $K$., Nojimo K.: Clinical characteristics of myocardial infarction following kawasaki disease: report of 11 cases. J Pediatr 1986; 108: 198-203. 